\title{
Peptide derived from desalinated boiled tuna extract inhibits adipogenesis through the downregulation of C/EBP- $\alpha$ and PPAR- $\gamma$ in 3T3-L1 adipocytes
}

\author{
YOUNG-MIN KIM, EUN-YOUNG KIM, IN-HYE KIM and TAEK-JEONG NAM \\ Department of Food Science and Nutrition, Pukyong National University, Nam-gu, Busan 608-737, Republic of Korea
}

Received December 1, 2014; Accepted March 2, 2015

DOI: $10.3892 / \mathrm{ijmm} .2015 .2127$

\begin{abstract}
Recently, obesity has increased due to a variety of reasons, including the availability of 'fast food' and high-fat diets. Developing anti-obesity functional drugs and foods from natural sources may offer solutions to this global concern. Generally, tuna is a high-protein, low-fat and low-calorie food with various bioactive effects. It may improve memory, reduce cholesterol levels and positively affect the development of brain cells. In this study, we screened the anti-obesity potential of peptides derived from tuna protein. We then observed protein bands by the Coomassie blue staining of a sodium dodecyl sulfate-polyacrylamide gel electrophoresis (SDS-PAGE) gel. The protein mixture was concentrated and desalted using in-gel trypsin digestion and a C18 nano column and POROS R2 reversed-phase preparation, prior to quadrupole time-of-flight mass spectrometry (Q-TOF MS/MS). We screened the peptides for their ability to affect adipogenesis in 3T3-L1 adipocytes. We also measured glucose uptake, triglyceride levels and lipid droplets using Oil Red O staining. As a result, we confirmed that one peptide inhibited adipocyte differentiation. We also observed the expression of obesity-related genes by western blot analysis and reverse transcription-polymerase chain reaction. The peptide from the tuna extract significantly reduced the expression levels of CCAAT/enhancer-binding protein $\alpha$ $(\mathrm{C} / \mathrm{EBP}-\alpha)$ and peroxisome proliferator-activated receptor- $\gamma$ (PPAR- $\gamma$ ) adipocyte marker genes. Thus, our data suggest that this peptide from boiled tuna extract reduces lipid components and adipogenesis in 3T3-L1 cells, and these characteristics may be of value in the development of anti-obesity foods.
\end{abstract}

\section{Introduction}

Obesity involves the adjustment of metabolism for energy storage and the accumulation of fat (1). The accumulation

Correspondence to: Professor Taek-Jeong Nam, Department of Food Science and Nutrition, Pukyong National University, 599-1 Daeyeon 3-Dong, Nam-gu, Busan 608-737, Republic of Korea

E-mail: namtj@pknu.ac.kr

Key words: CCAAT/enhancer-binding protein $\alpha$, 3T3-L1 adipocytes, peroxisome proliferator-activated receptor- $\gamma$, tuna peptide of excess fat is accompanied by inflammatory reactions that can induce chronic diseases, including diabetes, hypertension and cardiovascular disease $(2,3)$. Accordingly, there has been active research on stimulating metabolism or preventing the inflammatory response and excessive lipid accumulation in cells through lipid degradation.

Obesity has a variety of causes, including endocrine disorders and genetic factors. Recently, with the increase in the consumption of high-fat diets and the availability of 'fast food', increases in the obesity rate and, consequently, in the numbers of adult patients with various chronic diseases have been observed. With obesity, major concerns include the increased serum triglyceride and low-density lipoprotein (LDL)-cholesterol levels which then lead to increased cardiovascular diseases.

Hyperlipidemia can lead to a number of complications, including respiratory dysfunction, infertility and even certain thypes of cancer due to the accumulation of neutral lipids in peripheral tissues and the abdomen that can induce insulin resistance (4). Thus, avoiding this cascade of metabolic events has become a major concern worldwide, and functional foods with anti-obesity effects have been developed (5).

Tuna is a nutritionally superior high-protein food with various positive effects, including lowering the blood cholesterol levels and preventing arteriosclerosis, and is known to possess anticancer properties $(6,7)$. Boiled tuna extract contains various ingredients, including useful proteins derived from collagen and free amino acids (8). In this study, we confirmed the anti-obesity effects and the inhibitory effects on adipogenesis of a tuna-derived peptide in 3T3-L1 cells.

Generally, adipocytes play important roles in energy homeostasis and lipid metabolism. The differentiation of adipocytes from preadipocytes is indicative of clear morphological and biochemical changes (9). In vitro, 3T3-L1 cells, preadipocytes or matrix-derived precursor cells from adipose tissue can be used to demonstrate the differentiation process of adipocytes. The differentiation of adipocytes can be induced by the glucocorticoid, dexamethasone, and the phosphodiesterase inhibitor, methylisobutylxanthine. During this process, changes in cell shape occur, together with cell division and an adipocyte phenotype can be observed within 5-7 days (10). Adipocyte differentiation involves changes in the expression of various proteins. In particular, as previously demonstrated, the expression levels of proteins involved in the transport of 
lipids and hormonal responses related to lipid metabolism are affected (11). In addition, lipids have been shown to induce the accumulation of triglycerides (TG) and the upregulation of the transcription factors, CCAAT/enhancer-binding protein (C/EBP)- $\alpha$ and peroxisome proliferator-activated receptor- $\gamma$ (PPAR- $\gamma$ ) (12). IBMX and dexamethasone induce the upregulation of $\mathrm{C} / \mathrm{EBP}-\beta$ and $\mathrm{C} / \mathrm{EBP}-\delta$ during the early stages of adipocyte differentiation (13). Subsequently, during the later stages, C/EBP- $\alpha$ and PPAR- $\gamma$ induce the activation of adipocyte-specific mRNAs (14). In this study, we used various assays to assess the anti-obesity effects of a tuna peptide derived from desalinated boiled tuna extract on 3T3-L1 cells.

\section{Materials and methods}

Preparation of desalinated boiled tuna extract. The desalinated boiled tuna extract used in this study was prepared in Korea in 2014. First, boiled tuna extract was centrifuged to remove any suspended solids that may interfere with the desalting step. This process involves a change from 55 Brix, 13\% salinity to 45 Brix, $12 \%$ salinity. We performed membrane filtration (membrane 2319/size $200 \mathrm{kDa}$ ) on the desalinated boiled tuna extract. We finally obtained a tuna extract of 30 Brix, $1 \%$ salinity, which was subjected to heat exchanger-type momentary sterilization (conditions: $110^{\circ} \mathrm{C}, 10 \mathrm{sec}$ ). The tuna extract sample was then divided into $1.5-\mathrm{ml}$ tubes and stored at $-70^{\circ} \mathrm{C}$ until use.

Preparation of soluble/insoluble boiled tuna proteins and synthesis of tuna peptides. First, we reacted the desalinated boiled tuna extract with Tween-20 and -60 extraction buffer by overnight incubation at room temperature. The boiled tuna extract in extraction buffer was centrifuged $(5,000 \mathrm{rpm}$, $10 \mathrm{~min}, 4^{\circ} \mathrm{C}$ ) and the upper phase was collected and mixed with cold methanol and chloroform to separate the proteins. The mixture was centrifuged again $\left(12,000 \mathrm{rpm}, 5 \mathrm{~min}, 4^{\circ} \mathrm{C}\right)$, and the aqueous layer was removed and cold methanol was added, followed by further centrifugation $\left(12,000 \mathrm{rpm}, 10 \mathrm{~min}, 4^{\circ} \mathrm{C}\right)$. Finally, we removed the supernatant and dried the pellet.

We assessed the molecular weight of the tuna proteins by Coomassie blue staining. After subjecting the tuna extract to sodium dodecyl sulfate-polyacrylamide gel electrophoresis (SDS-PAGE), the protein bands were observed on the gel using staining solution (7\% acetic acid, $40 \%$ methanol, $0.1 \%$ Coomassie blue) and destaining solution (7\% acetic acid, $20 \%$ methanol). Tuna extract proteins of $\sim 10 \mathrm{kDa}$ were selected for analysis by quadrupole time-of-flight mass spectrometry (Q-TOF MS/MS). Each protein sample was subjected to in-gel trypsin digestion according to the method described in the study by Shevchenko et al (15). The digested peptide mixture was desalted and concentrated, then prepared using a C18 nano column and POROS R 2 reverse-phase material (20-30- $\mu \mathrm{m}$ bead size; PerSeptive Biosystems, Framingham, MA, USA). MS/MS analysis was performed using a nano-ESI and MicrOTOF-Q mass spectrometer (Bruker Daltonics, Bremen, Germany). Following their identification, 3 peptides were synthesized by Peptron (Daejeon, Korea): tuna-derived peptide, D-I-V-D-KI-E-I (TP-D), tuna-derived peptide, I-D-T-I-I-E-T-I-M-E (TP-I) and tuna-derived peptide, N-I-N-E-D-P-Y-E-N-W-I-V (TP-N). Purification of the tuna-derived peptides was performed using a C18 column (Shiseido CAPCELL PAK; Shiseido, Tokyo,
Japan) and a Shimadzu Prominence high-performance liquid chromatography (HPLC) apparatus in $0.1 \%$ trifluoroacetic acid (TFA)/water and a gradient of $0-90 \%$ acetonitrile in $0.1 \%$ TFA, with a flow rate of $1 \mathrm{ml} / \mathrm{min}$ and UV detection at $220 \mathrm{~nm}$. The TP-D, TP-I and TP-N molecular weights were determined as 944, 1,177 and 1,505 kDa, respectively, by mass analysis (HP 1100 series LC/MSD).

Cell culture and adipocyte differentiation. The 3T3-L1 mouse fibroblasts (American Type Culture Collection, Manassas, VA, USA) were maintained at $37^{\circ} \mathrm{C}$ in a $5 \% \mathrm{CO}_{2}$, humidified atmosphere. The cells were cultured in Dulbecco's modified Eagle's medium (DMEM) with $10 \%$ bovine calf serum (BCS; HyClone, Logan, UT, USA) and $100 \mathrm{U} / \mathrm{ml}$ penicillin $/ 100 \mathrm{mg} / \mathrm{ml}$ streptomycin. The 3T3-L1 cells were cultured to $\sim 60-80 \%$ confluence in a 6-well plate, and upon confluence, were allowed to grow for 2-4 days in DMEM medium containing $10 \%$ fetal bovine serum (FBS; HyClone). Cell differentiation was initiated by treatment with MDI $(0.5 \mathrm{mM}$ IBMX, $0.25 \mu \mathrm{M}$ dexamethasone and $10 \mathrm{mg} / \mathrm{l}$ insulin) for $48 \mathrm{~h}$. The medium was then replaced with DMEM supplemented with $10 \mathrm{mg} / \mathrm{l}$ insulin and changed once every 2 days.

Glucose uptake assay. The 3T3-L1 preadipocytes were incubated with DMEM containing 10\% BCS. Cell differentiation was induced by treatment with MDI in fresh DMEM containing $10 \%$ FBS. Following differentiation, the medium was changed to serum-free medium (SFM), and the cells were treated with TP-D, TP-I or TP-N at $500 \mathrm{ng} / \mathrm{ml}$ for $48 \mathrm{~h}$ before the glucose uptake assay. After collecting the cell culture medium, we confirmed glucose uptake using a kit according to the manufacturer's instructions (Asan Pharmaceutical Co., Ltd., Gyeonggi, Korea). Enzyme solution was added to the culture medium and maintained at $37^{\circ} \mathrm{C}$ for $5 \mathrm{~min}$ in a $5 \% \mathrm{CO}_{2}$, humidified atmosphere. The absorbance at $500 \mathrm{~nm}$ was measured within $40 \mathrm{~min}$. The absorbance of the solution in each well was measured at $490 \mathrm{~nm}$ using a microplate reader (Benchmark microplate reader; Bio-Rad Laboratories, Hercules, CA, USA).

TG component assay. The 3T3-L1 preadipocytes were incubated with DMEM containing 10\% BCS. Cell differentiation was induced by MDI treatment in fresh DMEM containing $10 \%$ FBS. After differentiation, the medium was changed to SFM supplemented with $500 \mathrm{ng} / \mathrm{ml}$ TP-D, TP-I and TP-N for $48 \mathrm{~h}$ prior to the TG assay. We performed the TG assay according to the kit protocol (Cleantech TG-S kit; Asan Pharm Co., Ltd., Seoul, Korea). The enzyme solution was added to the cell lysate and maintained at $37^{\circ} \mathrm{C}$ for $10 \mathrm{~min}$ in a $5 \% \mathrm{CO}_{2}$, humidified atmosphere. The absorbance at $550 \mathrm{~nm}$ was measured within $60 \mathrm{~min}$. The absorbance of the solution in each well was measured at $490 \mathrm{~nm}$ using a microplate reader (Benchmark microplate reader; Bio-Rad Laboratories).

Oil Red $O$ staining. The 3T3-L1 cells were washed carefully with phosphate-buffered saline (PBS) and then fixed with $10 \%$ formalin for $5 \mathrm{~min}$. The formalin was then refreshed followed by incubation for $1 \mathrm{~h}$. After removal of the formalin, $60 \%$ isopropanol was added to each well and dried. Subsequently, 60\% Oil Red O staining solution was added to each well for $1 \mathrm{~h}$. In the final step, the wells were washed 
Table I. Oligonucleotide sequences of the primer pairs used for RT-PCR.

\begin{tabular}{ll}
\hline Name & \multicolumn{1}{c}{ Sequences of primers } \\
\hline $\begin{array}{l}\text { C/EBP- } \alpha \\
\text { Sense }\end{array}$ & 5'-CTG-CCC-CTC-AGT-CCC-TGT-C-3' \\
Antisense & 5'-GTT-CCT-TCA-GCA-ACA-GCG-G-3' \\
PPAR- $\gamma$ & \\
Sense & 5'-CCT-GTT-GAC-CCA-GAG-CAT-GG-3' \\
Antisense & 5'-CGA-GTG-GTC-TTC-CAT-CAC-GC-3' \\
GAPDH & \\
Sense & 5'-AAC-TTT-GGC-ATT-GTG-AAG-G3' \\
Antisense & 5'-ACA-CAT-TGG-GGG-TAG-GAA-CA-3' \\
\hline $\begin{array}{l}\text { RT-PCR, reverse transcription-polymerase chain reaction; GAPDH, } \\
\text { glyceraldehyde } \\
\text { proliferator-activated receptor- } \gamma \text {; C/EBP- } \alpha, \text { CCAAT/enhancer-binding } \\
\text { protein } \alpha .\end{array}$
\end{tabular}

3 times with PBS, and the cell morphology and staining of the lipid droplets were observed using an inverted microscope (ECLIPSE TS100-F; Nikon, Tokyo, Japan).

Reverse transcription-polymerase chain reaction ( $R T-P C R)$ for the expression of $m R N A s$. The 3T3-L1 preadipocytes were seeded into 6 -well plates at $2 \times 10^{4}$ cells/well in $2 \mathrm{ml}$ medium. Cell differentiation was induced by treatment with MDI in fresh DMEM containing 10\% FBS. Following differentiation, the medium was changed to SFM supplemented with 500 or $1,000 \mathrm{ng} / \mathrm{ml}$ TP-D for $48 \mathrm{~h}$. The cells were then treated with TRIzol reagent (Invitrogen, Carlsbad, CA, USA), and the extracted RNA was used as a template for cDNA synthesis using an oligo(dT) primer (Intron Co., Gyeonggi, Korea). The synthesized cDNA was mixed with 2X TOPsimple DyeMIX-nTaq (Enzynomics Inc., Daejeon, Korea) and primers (Table I) in $0.1 \%$ diethylpyrocarbonate (DEPC)-treated water for PCR. Using a $1 \%$ agarose gel, the PCR products were separated and stained with Red safe nucleic acid staining solution (Intron Co.).

Western blot analysis. The 3T3-L1 preadipocytes were seeded into 6 -well plates at $2 \times 10^{4}$ cells/well in $2 \mathrm{ml}$ medium. Cell differentiation was induced by treatment with MDI in fresh DMEM containing 10\% FBS. Following differentiation, the medium was changed to SFM supplemented with 500 or $1,000 \mathrm{ng} / \mathrm{ml}$ TP-D for $48 \mathrm{~h}$. The collected cells were washed with PBS, and extraction lysis buffer (20 mM Tris-base, $\mathrm{pH} 8$, $150 \mathrm{mM} \mathrm{NaCl}, 100 \mu \mathrm{M}$ sodium vanadate, $100 \mu \mathrm{M}$ ammonium molybdate, $10 \%$ glycerol, $0.1 \%$ Nonidet P-40, $0.1 \%$ SDS, $1 \mathrm{mM}$ glycerophosphate, $1 \mu \mathrm{g} / \mathrm{ml}$ aprotinin, $1 \mu \mathrm{g} / \mathrm{ml}$ leupeptin, $1 \mu \mathrm{g} / \mathrm{ml}$ pepstatin A and $1 \mathrm{mM}$ PMSF) was added. The proteins were separated on 7-15\% SDS-PAGE gels and transferred onto polyvinylidene fluoride membranes (Millipore, Billerica, MA, USA). The membranes were blocked at room temperature with $1 \%$ bovine serum albumin in TBS-T $(10 \mathrm{mM}$ Tris- $\mathrm{HCl}$, $\mathrm{pH} 7.5,150 \mathrm{mM} \mathrm{NaCl}$ and $0.1 \%$ Tween-20) and then incubated

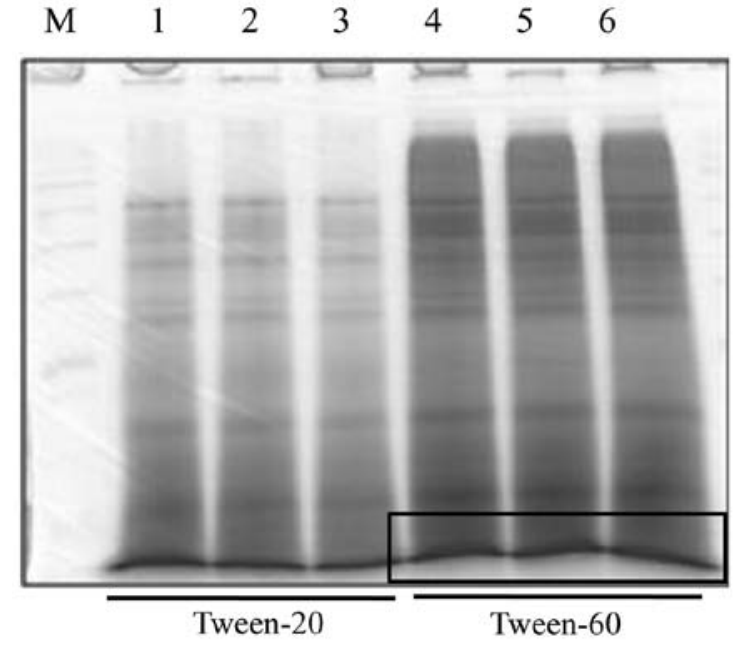

Figure 1. Profile map of the proteins separated from desalinated boiled tuna extract. The proteins from desalinated boiled tuna extract and the sample were subjected to sodium dodecyl sulfate-polyacrylamide gel electrophoresis (SDS-PAGE), and the gels were stained with Coomassie blue. M, Marker; 1-3, extract using Tween-20 buffer; 4-6, extract using Tween-60 buffer.

with primary antibodies: anti-C/EBP- $\alpha$ (1:1,000; sc-9314), anti-PPAR- $\gamma(1: 1,000 ;$ sc-1984) and anti-GAPDH $(1: 1,000$; sc-25778) (Santa Cruz Biotechnology, Inc., Santa Cruz, CA, USA). The secondary antibody was a peroxidase-conjugated goat (sc-2420), mouse, or rabbit antibody (sc-358920;f 1:10,000; GE Healthcare Bio-Sciences, Piscataway, NJ, USA). The signal was developed using SuperSignal West Pico Stable Peroxide Solution and the SuperSignal West Pico Luminol/Enhancer solution (Thermo Fisher Scientific, Rockford, IL, USA) prior to exposure to X-ray film (Kodak, Rochester, NY, USA).

Statistical analysis. The results are expressed as the means \pm SD. SPSS software (version 10.0; SPSS, Inc., Chicago, IL, USA) was used. Comparisons were made using ANOVA and Duncan's multiple range test. The level of significance was set at $\mathrm{P}<0.05$.

\section{Results}

Soluble/insoluble boiled tuna protein and synthetic tuna peptides. In this study, we performed a desalting process before ultimately extracting the tuna peptides. We confirmed the molecular weight of the tuna proteins by Coomassie blue staining. We observed a separated protein of boiled tuna extract at $\sim 10 \mathrm{kDa}$ and performed protein extraction using Tween-60 extraction buffer, as it yielded larger quantities of protein than did Tween-20 buffer (Fig. 1). We then characterized the $\sim 10-\mathrm{kDa}$ protein band by Q-TOF MS/MS. As shown in Fig. 2, we determined 3 peptide peaks and sequenced these tuna-derived peptides. These samples were termed tuna-derived peptide, D-I-V-D-K-I-E-I (TP-D), tuna peptide, I-D-T-I-I-E-T-I-M-E (TP-I) and tuna peptide, N-I-N-E-D-P-YE-N-W-I-V (TP-N); their molecular weights were determined to be $944,1,177$ and $1,505 \mathrm{kDa}$, respectively (Table II).

Effects of TP-D, TP-I and TP-N on glucose uptake in 3T3-LI adipocytes. Glucose consumption is a prerequisite for 3T3-L1 cell differentiation. In this study, we compared glucose 
Table II. Identification analysis of the $10-\mathrm{kDa}$ peptide among the boiled tuna proteins.

$\begin{array}{ll}\text { Identification } & \text { Peptide }\end{array}$

Glycine cleavage system $\mathrm{H}$ protein $\mathrm{OS}=$ Clostridium

Carboxidivorans P7
TP-D: DI(L)VDKI(L)EI(L)

TP-I: I(L)DTI(L)I(L)ETI(L)ME

TP-N: NI(L)NEDPYENWI(L)V

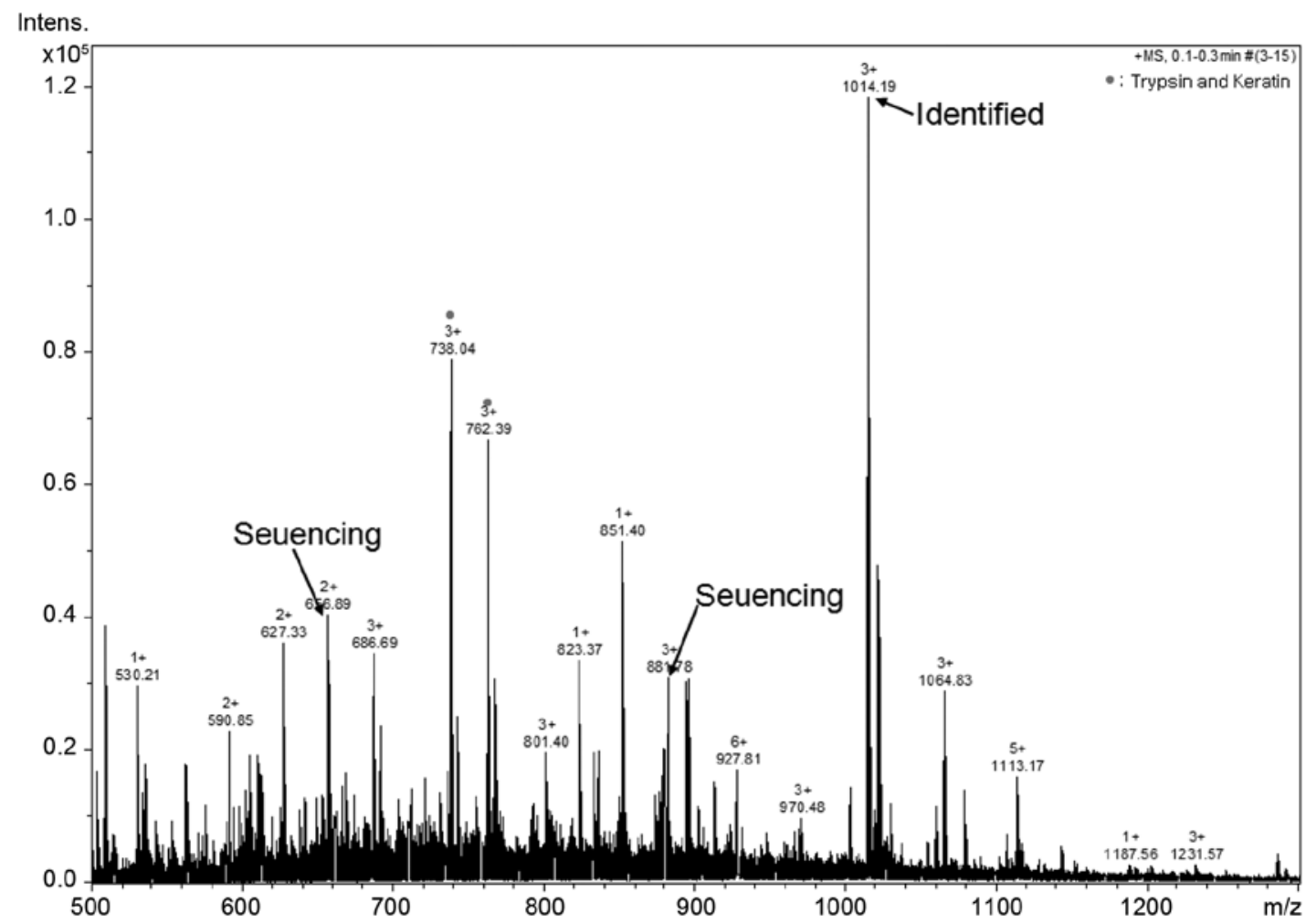

Figure 2. Quadrupole time-of-flight mass spectrometry (Q-TOF MS/MS) of the 10-kDa protein from the desalinated boiled tuna extract. The extract was desalted and concentrated using a C18 nano column and POROS R2 reverse-phase material. Identification analysis of the 10-kDa protein band using nano-ESI and a MicrOTOF-Q mass spectrometer showed 3 peptide peaks.

consumption, measured by a glucose uptake assay, between the differentiated cell group (MDI treatment) and the groups of cells treated with TP-D, TP-I or TP-N. All 3 tuna peptides were applied at concentrations of 500 and $1,000 \mathrm{ng} / \mathrm{ml}$ for $48 \mathrm{~h}$. The TP-D-treated cells showed decreased glucose uptake compared with the MDI-treated group. TP-D significantly attenuated glucose uptake following treatment for $48 \mathrm{~h}$ at both 500 and $1,000 \mathrm{ng} / \mathrm{ml}$ with a maximal effect observed at the dose of $1,000 \mathrm{ng} / \mathrm{ml}$. However, TP-I and TP-N had no significant effect following treatment for $48 \mathrm{~h}$ at either of the concentrations used (Fig. 3).

Effects of TP-D, TP-I and TP-N on TG levels in 3T3-L1 adipocytes. In order to evaluate the effects of TP-D, TP-N and TP-I on TG levels in differentiating 3T3-L1 cells, the cells were treated with the tuna-derived peptides at the dose of 500 and $1,000 \mathrm{ng} / \mathrm{ml}$. Glucose consumption induced the active differentiation of the 3T3-L1 cells and the accumulation of TG. In the glucose uptake assay, we observed decreased glucose levels following treatment with TP-D. Thus, we performed a TG assay under the same TP-D, TP-N and TP-I treatment conditions. The TG levels in the 3T3-L1 cells treated with TP-D (at 500 or $1,000 \mathrm{ng} / \mathrm{ml})$, were significantly deceased in a dose-dependent manner (Fig. 4). However, the TG levels in the cells treated with TP-N and TP-I were not altered. Thus, all further experiments were performed using treatment with TP-D at concentrations of 500 and $1,000 \mathrm{ng} / \mathrm{ml}$ for $48 \mathrm{~h}$.

Effect of TP-D on lipid accumulation in 3T3-L1 adipocytes. In the previous assays, we observed a decrease in the glucose and TG levels by TP-D in a dose-dependent manner. Thus, we then wished to confirm the reduced lipid accumulation by Oil Red O staining, which targets lipid droplets and allows the visualization of lipids (16). To determine the effects of TP-D on lipid accumulation in differentiating 3T3-L1 cells, the cells were treated with 500 and 1,000 ng/ml of TP-D. The cells were stained with Oil Red O solution and examined under a microscope. The number of stained lipid droplets detected decreased following treatment with TP-D in a dose-dependent manner compared with the MDI-treated group (Fig. 5). 


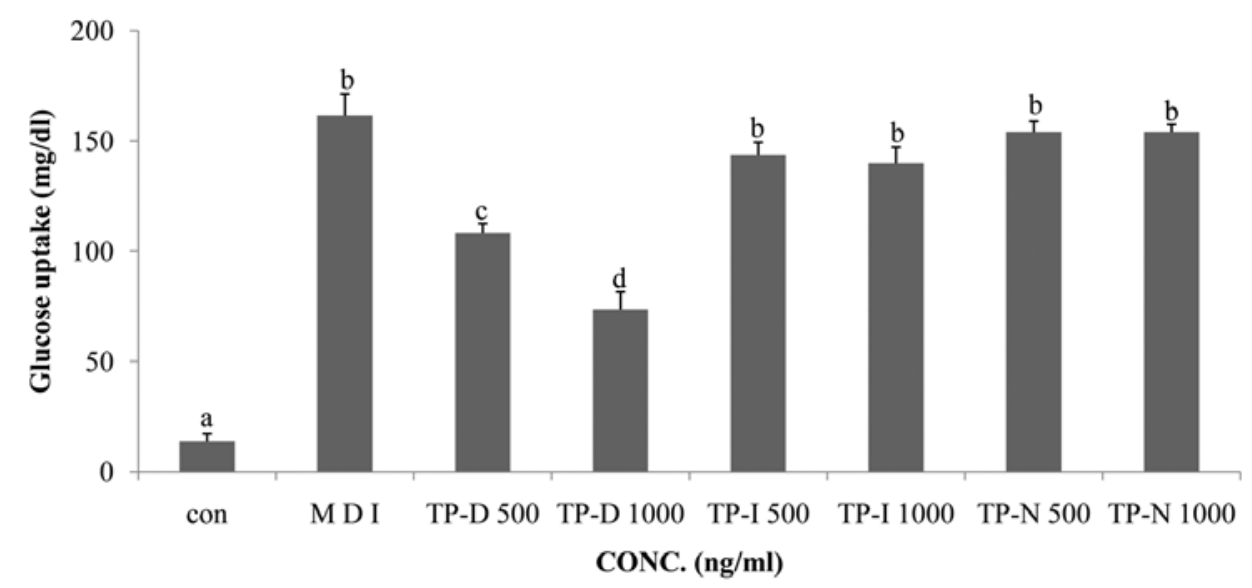

Figure 3. Effects of the tuna-derived peptides, TP-D, TP-I and TP-N, on glucose uptake by differentiated 3T3-L1 cells. 3T3-L1 cells were treated with TP-D, TP-I and TP-N at various concentrations $(500-1,000 \mathrm{ng} / \mathrm{ml})$ for $48 \mathrm{~h}$. The cell culture medium was assessed using a glucose uptake assay and an ELISA reader. Values represent the means $\pm \mathrm{SD} ; \mathrm{P}<0.05$ shown by ANOVA. Bars marked with different letters indicate a significant difference according to Duncan's multiple range test. con, control (no treatment); CONC., concentration.

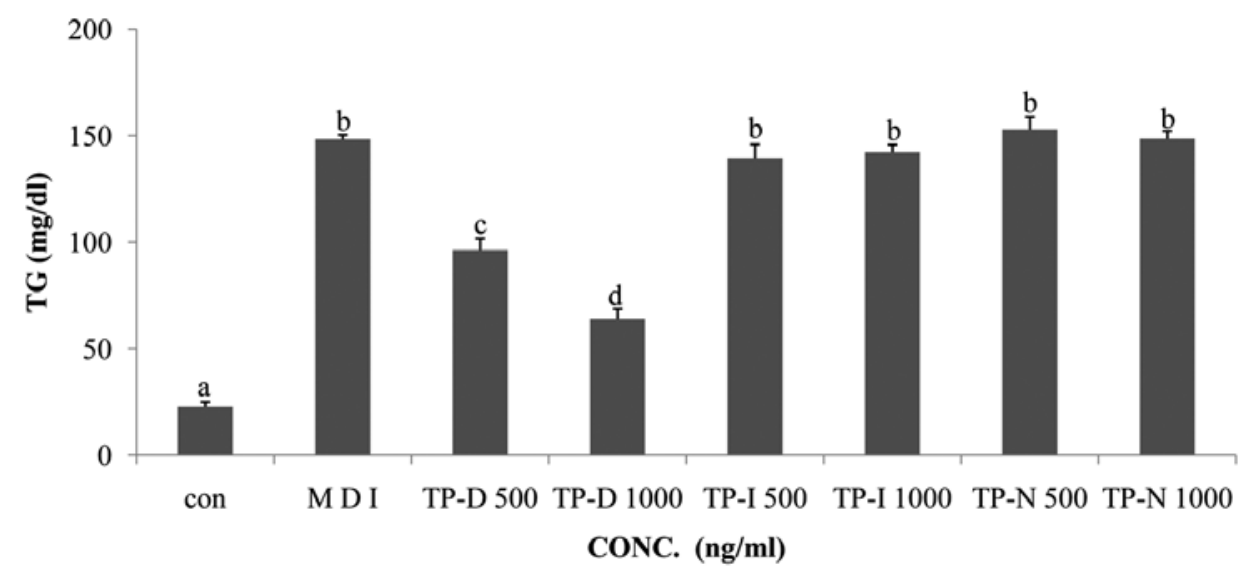

Figure 4. Effects of the tuna-derived peptides, TP-D, TP-I and TP-N, on triglyceride (TG) levels in differentiated 3T3-L1 cells. 3T3-L1 cells were treated with TP-D, TP-I and TP-N at various concentrations $(500-1,000 \mathrm{ng} / \mathrm{ml})$ for $48 \mathrm{~h}$. Cell lysates were assessed using a TG assay and an ELISA reader. Values represent the means $\pm \mathrm{SD} ; \mathrm{P}<0.05$ shown by ANOVA. Bars marked with different letters indicate a significant difference according to Duncan's multiple range test. con, control (no treatment); CONC., concentration.

Effects of TP-D on the expression of adipogenic genes $(C / E B P-\alpha$ and PPAR- $\gamma)$ during the differentiation of 3T3-LI adipocytes. The apparent morphological changes included changes in the number of lipid droplets and lipid accumulation during the differentiation of the cells into adipocytes. In addition, biochemical changes were observed in terms of the expression of adipocyte-specific protein markers. Generally, there is a significantly increased expression of transcription factors and the secretion of adipokines during adipocyte differentiation $(17,18)$. Among these transcription factors, C/EBP- $\alpha$ and PPAR $-\gamma$ are considered important (19), with increased expression levels observed in differentiated adipocytes. Their expression is induced during early differentiation, and during the later stages of differentiation, they induce the expression of various adipogenic genes. In our previous experiment, we confirmed the inhibition of lipid accumulation and cellular differentiation by treatment with TP-D. Thus, we then measured adipocyte-related factors at the protein and mRNA level by western blot analysis and RT-PCR, respectively, and observed the inhibition of the activation of $\mathrm{C} / \mathrm{EBP}-\alpha$ and PPAR- $\gamma$ proteins. In turn, the controlled inhibition of C/EBP- $\alpha$ and PPAR- $\gamma$ by TP-D inhibited adipocyte formation in a dose-dependent manner (Fig. 6).

\section{Discussion}

Recently, an increase in obesity has led to a concomitant increase in serious disorders, such as metabolic disease, hypertension, atherosclerosis and irregular eating habits $(20,21)$. Adipocytes play an important role in the balance of energy and in the regulation lipid metabolism and are associated with obesity and adipose tissue mass. Indeed, lipid accumulation during adipogenesis and the programmed differentiation of preadipocytes involve several steps related to obesity (22). A number of research studies have focused on reducing obesity by inhibiting lipogenesis and the differentiation of preadipocytes. 


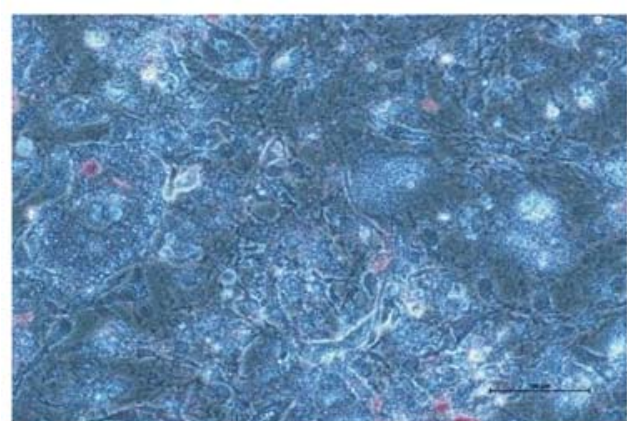

con

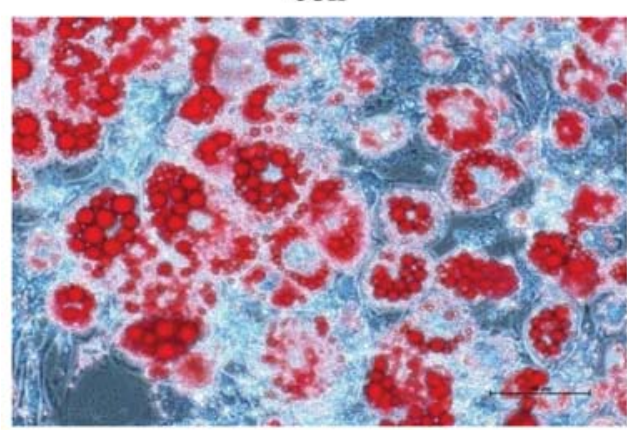

MDI-2D, Insulin + TP-D $500 \mathrm{ng} / \mathrm{ml}$

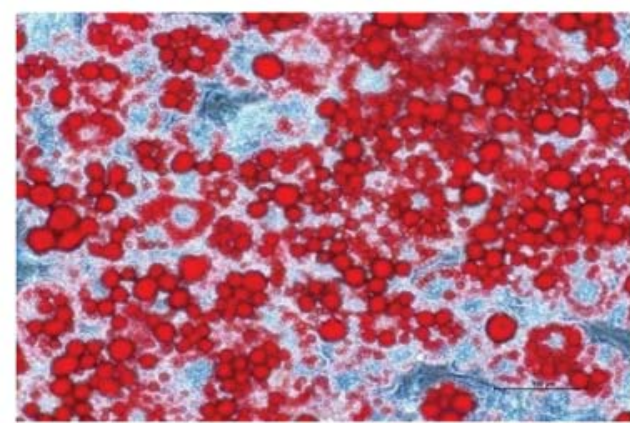

MDI-2D, Insulin

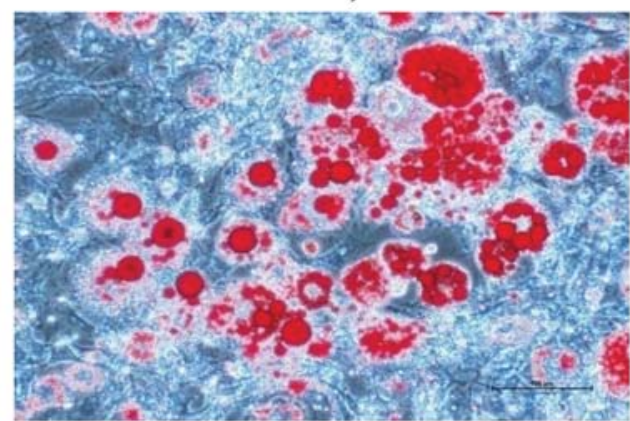

MDI-2D, Insulin + TP-D $1000 \mathrm{ng} / \mathrm{ml}$

Figure 5. Effects of the tuna-derived peptide, TP-D, on lipid accumulation in 3T3-L1 cells. 3T3-L1 cells were treated with TP-D at various concentrations (500-1,000 ng/ml) for $48 \mathrm{~h}$. Lipid droplets were assessed by Oil Red O staining. con, control (no treatment).

A
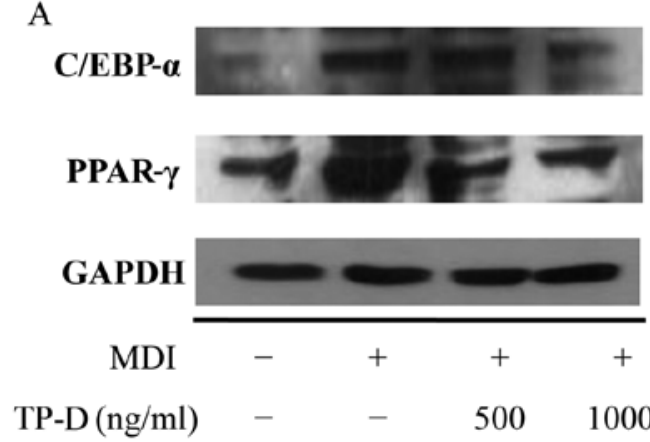

B

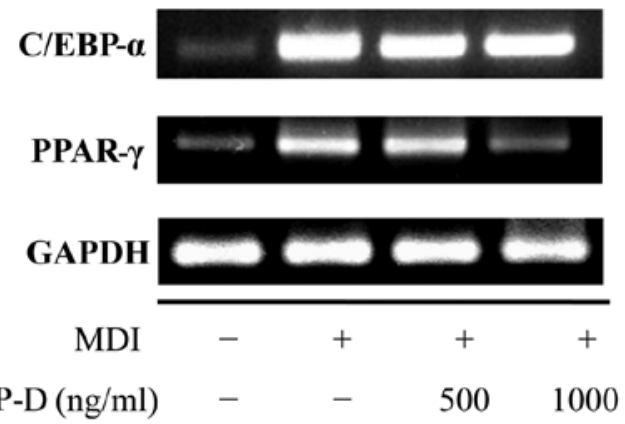

Figure 6. Inhibition of CCAAT/enhancer-binding protein $\alpha(\mathrm{C} / \mathrm{EBP}-\alpha)$ and peroxisome proliferator-activated receptor- $\gamma(\mathrm{PPAR}-\gamma)$ in 3T3-L1 cells treated with TP-D (500-1,000 ng/ml) for $48 \mathrm{~h}$. (A) Protein expression levels were measured by western blot analysis. (B) cDNA was subjected to reverse transcriptionpolymerase chain reaction (RT-PCR) for the analysis of mRNA expression by electrophoresis using a 1\% agarose gel stained with Red safe nucleic acid staining solution.

Additionally, research on and the development of 'functional foods' have been actively pursued to address obesity and its complications.

Tuna is a high-protein and nutritionally superior food. It exerts inhibitory effects on atherosclerosis by decreasing the serum cholesterol concentrations. It also has a wide range of bioactivities, including anticancer properties (23). The tuna catch is approximately 4 million tons per year worldwide, and $40 \%$ of that is processed into canned tuna (24). Tuna canning requires a boiling treatment, and 27,000 tons of boiled tuna byproduct result from this process (25). Boiled tuna contains various active ingredients, such as large amounts of free amino acids, collagen protein, carnosine and taurine. However, the study of its composition is incomplete, due to the difficulties in desalinating it and removing the coloration.
In this study, we separated peptides from boiled tuna and confirmed their anti-obesity properties on 3T3-L1 cells by the inhibition of their differentiation. 3T3-L1 cells are commonly used as a model for adipocyte differentiation to examine signaling pathways in adipogenesis (26). To examine the effects of the tuna peptides on the differentiation of adipocytes, 3T3-L1 preadipocytes were treated with MDI to induce differentiation and then cultured in medium containing insulin. The 3T3-L1 adipocytes were then treated with 500 or $1,000 \mathrm{ng} / \mathrm{ml} \mathrm{TP}-\mathrm{D}$, TP-I or TP-N for 2 days. We assessed the potentiating effects of TP-D, TP-I and TP-N on glucose uptake in 3T3-L1 adipocytes. Generally, a large amount of glucose consumption is required for the differentiation of 3T3-L1 preadipocytes into adipocytes. Treatment with 500 and 1,000 ng/ml TP-D led to a decrease in glucose consumption, by $\sim 33$ and $63 \%$, respectively, compared 
with the MDI group. However, treatment with TP-I and TP-N did not have any significant effects (Fig. 3). Effects on lipid accumulation were also observed. When the differentiation of the preadipocytes was induced, the consumption of glucose was increased. Thus, we measured the TG levels under the same treatment conditions used for the glucose assay. As a result, treatment with 500 and 1,000 ng/ml TP-D led to a decrease in TG levels by $\sim 35$ and $57 \%$, compared with the MDI-treated group. Treatment with TP-I and TP-N had no significant effects (Fig. 4). Based on these results, treatment with TP-D decreased the amount of lipid droplets in a dose-dependent manner compared with the MDI-treated group, as revealed by Oil Red $\mathrm{O}$ staining and the microscopy examination of the differentiated adipocytes. The stained lipid droplets showed a significantly reduced lipid accumulation in the differentiated adipocytes following treatment with TP-D in a dose-dependent manner (Fig. 5). Subsequently, we investigated the effects of TP-D on the differentiation of 3T3-L1 adipocytes by examining the expression levels of master adipogenic transcription factors, such as C/EBP- $\alpha$ and PPAR- $\gamma$. Generally, the differentiation of 3T3-L1 preadipocytes into adipocytes involves a series of transcriptional processes. C/EBP- $\alpha$ plays important roles during the early stages of preadipocyte differentiation, and together with the induced upregulation of PPAR- $\gamma$, these transcription factors activate a number of additional adipocyte genes directly $(27,28)$. PPAR- $\gamma$ promotes the expression of specific lipogenic and adipogenic genes during preadipocyte differentiation and is necessary and sufficient for fat formation (29). Thus, the downregulation of C/EBP- $\alpha$ and PPAR- $\gamma$ inhibits lipid accumulation and suppresses adipocyte differentiation. The expression levels of C/EBP- $\alpha$ and PPAR- $\gamma$ were examined by western blot analysis and RT-PCR. As a result, we found that TP-D induced the downregulation of C/EBP- $\alpha$ and PPAR- $\gamma$ (Fig. 6) and inhibited the differentiation of 3T3-L1 cells, suggesting the potential use of TP-D in anti-obesity functional foods and/or therapeutics.

\section{Acknowledgements}

This study was a part of the project entitled 'Functional materials and foods using fisheries by-products', funded by the Ministry of Oceans and Fisheries, Korea (20130279).

\section{References}

1. Kim DM, Choi HR, Park A, Shin SM, Bae KH, Lee SC, Kim IC and Kim WK: Retinoic acid inhibits adipogenesis via activation of Wnt signaling pathway in 3T3-L1 preadipocytes. Biochem Biophys Res Commun 434: 455-459, 2013.

2. Ahn J, Lee H, Kim S and Ha T: Curcumin-induced suppression of adipogenic differentiation is accompanied by activation of Wnt/ $\beta$-catenin signaling. Am J Physiol Cell Physiol 298: C1510-C1516, 2010.

3. Kopelman PG: Obesity as a medical problem. Nature 404: 635-643, 2000.
4. Lew EA: Mortality and weight: Insured lives and the American Cancer Society studies. Ann Intern Med 103: 1024-1029, 1985.

5. Visscher TL and Seidell JC: The public health impact of obesity. Annu Rev Public Health 22: 355-375, 2001.

6. Carrill KK: Biological effects of fish oils in relation to chronic disease. Lipis 21: 731-732, 1986.

7. Mehta J, Lopez LM and Wargovich T: Eicosapentaenoic acid: Its relevance in atherosclerosis and coronary artery disease. Am J Cardiol 59: 155-159, 1987

8. Lee HS, Kim HJ, Choi JI, et al: Antioxidant activity of the ethanol extract from cooking drips of Thunnus thynnus by gamma irradiation. J Korean Soc Food Sci Nutr 37: 810-814, 2008.

9. Lee JA, Ahn EK, Hong SS and Oh JS: Anti-obesity effect of ethyl acetate extracts from Agrimonia pilosa Ledeb. in 3T3-L1 preadipocytes. J Korean Soc Food Sci Nutr 41: 161-167, 2012.

10. Smas CM and Sul HS: Control of adipocyte differentiation. Biochem J 309: 697-710, 1995.

11. Carey M: The enhanceosome and transcriptional synergy. Cell 92: 5-8, 1998

12. Darlington GJ, Ross SE and MacDougald OA: The role of C/EBP genes in adipocyte differentiation. J Biol Chem 273: 30057-30060, 1998.

13. Brun RP, Kim JB, Hu E, Altiok S and Spiegelman BM: Adipocyte differentiation: A transcriptional regulatory cascade. Curr Opin Cell Biol 8: 826-832, 1996 .

14. Hu E, Tontonoz P and Spiegelman BM: Transdifferentiation of myoblasts by the adipogenic transcription factors PPAR gamma and C/EBP alpha. Proc Natl Acad Sci USA 92: 9856-9860, 1995.

15. Shevchenko A, Wilm M, Vorm O and Mann M: Mass spectrometric sequencing of proteins silver-stained polyacrylamide gels. Anal Chem 68: 850-858, 1996.

16. Beaudoin A: New technique for revealing latent fingerprints on wet, porous surfaces: Oil Red O. J Forensic Identif 54: 413-421, 2004.

17. Rosen ED, Walkey CJ, Puigserver P and Spiegelman BM: Transcriptional regulation of adipogenesis. Genes Dev 14: 1293-1307, 2000.

18. Jeon T, Hwang SG, Hirai S, Matsui T, Yano H, Kawada T, Lim BO and Park DK: Red yeast rice extracts suppress adipogenesis by down-regulating adipogenic transcription factors and gene expression in 3T3-L1 cells. Life Sci 75: 3195-3203, 2004.

19. Cornelius P, MacDougald OA and Lane MD: Regulation of adipocyte development. Annu Rev Nutr 14: 99-129, 1994.

20. Hursting SD and Hursting MJ: Growth signals, inflammation, and vascular perturbations: Mechanistic links between obesity, metabolic syndrome, and cancer. Arterioscler Thromb Vasc Biol 32: 1766-1770, 2012.

21. Kant R: Sweet proteins - potential replacement for artificial low calorie sweeteners. Nutr J 4: 5, 2005.

22. Unger RH and Zhou YT: Lipotoxicity of beta-cells in obesity and in other causes of fatty acid spillover. Diabetes 50 (Suppl 1): S118-S121, 2001.

23. Hunter E: PUFA and eicosanoid research. JAOCS 64: 1088, 1987.

24. Kang MK and Song KB: Quality characteristics of Gochujang with the addition of skipjack cooking broth as protein source. Korean J Food Preserv 13: 457-464, 2006.

25. Kim JS, Yeum DM, Kang HG, Kim IS, Kong CS, Lee TG and Heu MS: Fundamentals and applications for canned foods. Vol. 95. Hyoil Publishing Co., Seoul, Korea, pp351-360, 2002.

26. Green $\mathrm{H}$ and Kehinde O: An established preadipose cell line and its differentitation in culture. II. Factors affecting the adipose conversion. Cell 5: 19-27, 1975.

27. Lee $\mathrm{H}, \mathrm{Bae} \mathrm{S}$ and Yoon Y: The anti-adipogenic effects of (-)epigallocatechin gallate are dependent on the WNT/ $\beta$-catenin pathway. J Nutr Biochem 24: 1232-1240, 2013.

28. He Y, Li Y, Zhao T, Wang Y and Sun C: Ursolic acid inhibits adipogenesis in 3T3-L1 adipocytes through LKB1/AMPK pathway. PLoS One 8: e70135, 2013.

29. Farmer SR: Transcriptional control of adipocyte formation. Cell Metab 4: 263-273, 2006. 